\title{
EFECTO DE CULTIVOS HOSPEDEROS Y NO HOSPEDEROS SOBRE PROPÁGULOS MICORRÍCICOS ARBUSCULARES ${ }^{1}$
}

\author{
Claudia Castillo ${ }^{1}$, Ivonne Astroza ${ }^{1}$, Fernando Borie $^{2}$, Rosa Rubio ${ }^{2}$ \\ ${ }^{1}$ Universidad Católica de Temuco, Casilla 15-D, Temuco, Chile \\ ${ }^{2}$ Universidad de La Frontera, Casilla 54-D, Temuco, Chile \\ Correspondencia: ccastill@uct.cl
}

\section{Effect of the host and non host crops on arbuscular mycorrhizal propagules}

Keywords: oats, lupine, rapeseed, Andisol, Inceptisol.

\begin{abstract}
Recent field studies have shown that fungal spores decrease when non host plants are used as a pre-culture. The objective of this study was to evaluate how host plant like oats (Avena sativa L.), and non host, as lupine (Lupinus albus L.), and rapeseed (Brassica napus L.) can influence on arbuscular mycorrhizal fungi (AMF) propagules diversity and phosphatase activity when growing in an Andisol and an Inceptisol. The trial was conducted from September 2006 through March 2007 in greenhouse conditions using $5 \mathrm{~kg}$ pots with in a completely randomized design with four replicates. The number of AMF spores was higher in the Andisol than the Inceptisol and highest when using oats than lupine or raps as plant host. Oats also showed a large enrichment of morphotypes, whereas lupine and raps were poor. The phosphatase activity (P-ase) in Inceptisol was lower than in Andisol and between crops increased in the order oats $<$ rapeseed $<$ lupine; while significant differences between lupines with other crops were registred. Again, our results reinforce the hypothesis that the mycorrhizae and phosphatase activity are complementary mechanisms developed by plants for a better $\mathrm{P}$ acquisition.
\end{abstract}

\footnotetext{
${ }^{1}$ Trabajo presentado al Segundo Simposio Internacional Suelos, Ecología y Medio Ambiente. Sociedad Chilena
} de la Ciencia del Suelo. Universidad de La Frontera. 8 al 9 de noviembre de 2007. 
Palabras claves: avena, lupino, raps, Andisol, Inceptisol.

\section{RESUMEN}

Estudios recientes en campo han demostrado que las esporas fúngicas disminuyen cuando se utilizan como pre-cultivo no hospederos. El objetivo de este trabajo fue estudiar la influencia de un cultivo hospedero, avena (Avena sativa L.) y cultivos no hospederos, como lupino (Lupinus albus L.) y raps (Brassica napus L.) sobre los propágulos de hongos micorrícicos arbusculares (HMA) y diversidad fúngica junto con la actividad fosfatásica en un Andisol serie Temuco e Inceptisol serie Lumaco. El ensayo se realizó en condiciones de invernadero, desde septiembre de 2006 hasta marzo de 2007, utilizándose macetas de $5 \mathrm{~kg}$ con un diseño experimental completamente al azar con cuatro repeticiones. El número de esporas HMA fue mayor en el Andisol que en el Inceptisol y mayor cuando se usó avena como planta hospedera que lupino y raps. La avena también mostró una gran riqueza de morfotipos mientras que, en lupino y raps fue baja. La actividad fosfatásica en el Inceptisol fue menor que en el Andisol y entre cultivos aumentó en orden avena $<$ raps $<$ lupino; mientras que, se encontraron diferencias significativas entre lupino con los otros cultivos. Nuevamente, nuestros resultados refuerzan la hipótesis que las micorrizas y actividad fosfatásica son mecanismos complementarios que utiliza la planta para una mejor captación de fósforo.

\section{INTRODUCCIÓN}

Para disminuir la perturbación del suelo y la competencia entre plantas es muy importante el uso de herramientas biológicas que aseguren el establecimiento exitoso de las especies vegetales, entre ellas, se encuentran los microorganismos rizosféricos. Estos cumplen un rol preponderante por las variadas funciones que realizan (Heredia, 2003), desarrollando algunas interacciones benéficas, del tipo micorrizas, simbiosis que se establece entre ciertos hongos del suelo con las raíces de las plantas vasculares (Guadarrama et al., 2004). Los hongos, mediante extensas redes de micelio, mejoran la captación de nitrógeno $(\mathrm{N})$, calcio $(\mathrm{Ca})$, potasio $(\mathrm{K})$ y nutrientes poco móviles como fósforo $(\mathrm{P})$, cobre $(\mathrm{Cu})$ y zinc $(\mathrm{Zn})$ (Souchie et al., 2006) aumentando el área de absorción radical lo que mejora la resistencia de la planta a los estreses bióticos y abióticos como patógenos radicales, déficit hídrico (Augé, 2004), salinidad y fitotoxicidad por aluminio (Al) (Borie y Rubio, 1999) y manganeso (Mn) (Mendoza y Borie, 1998).
La presencia de los hongos micorrícicos arbusculares (HMA) en el suelo puede cambiar el balance competitivo entre los hospederos aminorando la intensidad (Allen y Allen, 1990), ya que al actuar como extensiones del sistema radical favorecen la parte epígea de la planta hospedera, optimizando el proceso fotosintético y por tanto, el crecimiento y supervivencia del vegetal (Eissenstat y Newman, 1990). Como consecuencia, los HMA influyen sobre la dinámica de las comunidades y en la composición de especies (Castillo, 2005). Sin embargo, no todas las asociaciones entre HMA-planta son compatibles, pudiendo algunos hongos beneficiar en mayor grado a un hospedero y adaptarse a determinadas condiciones edafoclimáticas evidenciando marcadas diferencias, no sólo estructurales, sino también funcionales, entre especies, e incluso morfotipos de una misma especie (Linderman y Davis, 2004). 
Los propágulos fúngicos constituidos por trozos de raíces colonizadas, esporas y segmentos de hifas, generalmente se encuentran concentrados en los primeros centímetros del suelo (Bellgard, 1993) pudiendo sobrevivir bajo diferentes condiciones ambientales (Reeves, 1985), incluso cuando cultivos de plantas no micotróficas se encuentran presentes en el agroecosistema, declinando con el tiempo su viabilidad (Jasper et al., 1993).

En la actualidad existen numerosos estudios relacionados con diferentes respuestas de hospederos a la inoculación con HMA, especialmente basados en aspectos nutricionales de la planta; sin embargo, se dispone de escasa información sobre especificidad hospedera. Mientras la mayoría de los HMA pueden asociarse con un amplio rango de hospederos, estudios más recientes sugieren que su asociación dependería de la especie de hospedero involucrada y de un cierto grado de compatibilidad relacionado a una cierta dependencia de la planta por algunas especies HMA (McGonigle y Fitter, 1990). Por lo tanto, la diversidad en las comunidades fúngicas sería consecuencia de interacciones ecológicas entre los individuos como también, de relaciones entre los individuos con su medio ambiente. La gran mayoría de los métodos propuestos para evaluar la diversidad fúngica se refieren a la diversidad dentro de las comunidades. El índice ecológico más sencillo es la riqueza específica basada en el número de especies presentes, sin considerar el valor de importancia de las mismas (Moreno, 2001) y el índice de diversidad de Shannon-Wiener (H') basado en el concepto de equidad. Por otra parte, los índices basados en la dominancia son parámetros inversos a la equidad y consideran la representatividad de las especies con mayor valor de importancia, sin evaluar la contribución del resto (Magurran, 1988).

Además de la simbiosis micorrícica, las plantas utilizan otras estrategias para la captación de nutrientes, especialmente $\mathrm{P}$, como la exudación de enzimas desde las raíces, entre ellas, las fosfatasas, que catalizan la hidrólisis de los ésteres y anhídridos del ácido fosfórico (Tabatabai, 1994), además de ser responsables de la mineralización del $\mathrm{P}$ orgánico y liberación de $\mathrm{P}$ disponible. $\mathrm{Su}$ actividad es mayor en la superficie de la raíz que en el resto del suelo y se incrementa con la deficiencia de P (Portilla et al., 1998).

En nuestro país existen escasos antecedentes sobre el efecto producido por la secuencia de rotación de cultivos sobre la actividad y desarrollo de los propágulos micorrícicos, los cuales al encontrarse más concentrados en el horizonte superficial del suelo, podrían ser afectados en la rotación por el establecimiento de cultivos no micotróficos, como lupino (Lupinus albus L.) o raps (Brassica napus L.).

El objetivo de este trabajo fue estudiar la influencia de un cultivo hospedero como avena (Avena sativa L.) y de dos cultivos no hospederos como lupino y raps sobre los propágulos micorrícicos (raíces colonizadas, esporas, micelio) y diversidad fúngica junto con la actividad fosfatásica en un Andisol y un Inceptisol de la Región de La Araucanía.

\section{MATERIALES Y MÉTODOS}

El ensayo se realizó en los invernaderos de la Escuela de Agronomía, en el Campus Norte de la Universidad Católica de Temuco entre septiembre de 2006 a marzo de 2007. Se utilizaron macetas de $5 \mathrm{~kg}$ de capacidad conteniendo un Andisol serie Temuco (Soil Survey Staff, 1996), de la localidad de Lautaro (38 $34^{\prime} \mathrm{S} ; 7^{\circ} 27^{\prime} \mathrm{O}$ ) procedente de un cultivo de papas y un Inceptisol serie Lumaco (Soil Survey Staff, 1996), de la localidad de Purén $\left(38^{\circ} 2^{\prime} \mathrm{S} ; 7^{\circ} 6^{\prime} \mathrm{O}\right)$ proveniente de una pradera natural, muestreados a $20 \mathrm{~cm}$ de profundidad (Cuadro 1). 
Cuadro 1. Características químicas del Andisol e Inceptisol.

Table 1. Chemical characteristics of an Andisol and Inceptisol.

\begin{tabular}{lcc}
\hline Variable & Andisol & Inceptisol \\
\hline $\mathrm{pH}$ & $5,79 \pm 0,09^{2}$ & $5,05 \pm 0,14$ \\
Humedad $(\%)$ & $29,0 \pm 0,1$ & $5,1 \pm 0,1$ \\
$\mathrm{D}_{\text {ap }}^{1}\left(\mathrm{~g} \mathrm{~mL}^{-1}\right)$ & $0,40 \pm 0,02$ & $0,98 \pm 0,05$ \\
$\mathrm{P}$ total $\left(\mathrm{mg} \mathrm{kg}^{-1}\right)$ & $3182 \pm 125$ & $1008 \pm 84$ \\
$\mathrm{Na}\left(\mathrm{cmol}_{+} \mathrm{kg}^{-1}\right)$ & $0,25 \pm 0,02$ & $0,24 \pm 0,03$ \\
$\mathrm{Fe}\left(\mathrm{mg} \mathrm{kg}^{-1}\right)$ & $12,71 \pm 0,65$ & $19,47 \pm 2,23$ \\
$\mathrm{Al}\left(\mathrm{mg} \mathrm{kg}^{-1}\right)$ & $16,10 \pm 2,86$ & $64,05 \pm 2,01$ \\
\hline
\end{tabular}

${ }^{1}$ Densidad aparente

${ }^{2}$ Desviación estándar

El suelo fue tamizado a $5 \mathrm{~mm}$. Los cultivos seleccionados fueron: como hospedero micorrícico A. sativa cv. Lord y como cultivos no hospederos L. albus cv. Rumbo y B. napus cv. Arthur sembrándose ocho, seis y cuatro semillas pregerminadas y posteriormente raleadas a seis, cuatro y dos plantas, respectivamente. Durante todo el ensayo las macetas se mantuvieron a capacidad de campo $(-33 \mathrm{kPa})$ y bajo condiciones controladas de temperatura con un valor promedio de $23^{\circ} \mathrm{C}$ y un fotoperíodo de $16 \mathrm{~h}$.

\section{Parámetros fúngicos}

Porcentaje de colonización en las raíces por hongos micorrícicos arbusculares (HMA). Se determinó sólo en el cultivo hospedero (A. sativa) utilizando el método del intercepto de líneas (Giovannetti y Mosse, 1980) para lo cual, se cortaron trozos de $1 \mathrm{~cm}$ de raíces frescas que se tiñeron con azul de tripán al 0,05 \% (Phillips y Hayman, 1970) observándose bajo el microscopio estereoscópico (40x) la presencia o ausencia de estructuras fúngicas (micelio, esporas o vesículas intraradicales).
Esporas HMA. Las esporas se extrajeron desde el suelo usando el método del tamizado húmedo y decantación, con posterior separación en gradiente de sacarosa al $70 \%$ y centrifugación según metodología descrita por Sieverding (1991). Finalmente, en placa de Doncaster se contabilizaron bajo lupa estereoscópica expresando el resultado en número de esporas $100 \mathrm{~mL}^{-1}$ de suelo seco.

Longitud de micelio total. Se determinó por extracción de las hifas desde el suelo utilizando glicerina ácida en caliente, de acuerdo con la técnica modificada para suelos volcánicos (Rubio et al., 2003). Luego, se tiñeron con azul de tripán y se cuantificaron según el método del intercepto de líneas (Giovannetti y Mosse, 1980).

Diversidad HMA. Las comunidades fúngicas fueron descritas mediante la riqueza (R), equidad (E) y los índices de diversidad de Shannon-Wiener (H') y Simpson (D) (Franke-Snyder et al., 2001). La riqueza (R) es una medida sencilla de la biodiversidad basada únicamente en el número total de especies que pertenecen a 
la comunidad. El H' mide el grado promedio de incertidumbre y puede predecir la especie a la cual pertenece un individuo escogido al azar en una población, mientras D manifiesta la probabilidad que dos individuos elegidos al azar en una muestra sean de la misma especie. Los estudios de diversidad HMA se realizaron mediante observación morfológica de las esporas agrupadas según su homogeneidad respecto de la forma, color, la apariencia de la pared externa, el tamaño de la espora y la presencia, forma y coloración de la conexión hifal.

\section{Parámetros edáficos}

El pH se midió potenciométricamente (Extech 321990) mediante una suspensión suelo:agua en relación 1:2,5. Para la densidad aparente $\left(\mathrm{D}_{\mathrm{ap}}\right)$ se utilizó el método del cilindro (Steubing et al., 2002). El fósforo disponible se analizó mediante extracción con $\mathrm{NaHCO}_{3}$ 0,5 $\mathrm{M}$ a pH 8,5 y posterior lectura espectrofotométrica a 700 $\mathrm{nm}$ (Olsen y Sommers, 1982). Para determinar fósforo total se realizó una destrucción con hipobromito de sodio según metodología propuesta por Dick y Tabatabai (1977). La actividad fosfatásica ácida se determinó usando $p$-nitrofenilfosfato de acuerdo al procedimiento descrito por Tabatabai y Bremner (1969) con modificaciones para suelos volcánicos con altos contenidos de MO informadas por Rubio et al. (1990) cuantificándose la cantidad de $p$-nitrofenol liberado espectrofotométricamente a $400 \mathrm{~nm}$. Finalmente, los micronutrientes se extrajeron con DTPA y para los cationes se usó $\mathrm{CH}_{3} \mathrm{COONH}_{4} \mathrm{pH}$ 7,0 mientras que, Al intercambiable se extrajo con $\mathrm{KCl}$ y lectura posterior en espectrofotómetro de absorción atómica (EAA) según metodología descrita por Sadzawka et al. (2000).

\section{Análisis estadístico}

Se utilizó un diseño experimental completamente al azar con cuatro repeticiones. En el tratamiento estadístico de los datos se verificó normalidad y posteriormente mediante transformación arcoseno, se realizó un análisis de varianza usando ANDEVA de una vía, seguido por el test de Tukey de rango múltiple $(\alpha=0,05)$. Para el análisis estadístico se utilizó el programa SPSS para Windows versión 13.0.

\section{RESULTADOS Y DISCUSIÓN}

En ambos suelos, se observó una disminución de los propágulos fúngicos en presencia de una planta no hospedera como lupino y raps en comparación con un hospedero, avena (Figura 1). Así, en el Andisol, el número de esporas HMA que permanecieron en el suelo fue significativamente diferente al comparar la avena con lupino, incrementando en $212 \%$ la cantidad de propágulos; sin embargo, entre avena y el otro cultivo no hospedero raps no se encontraron diferencias, pero avena incrementó en $39 \%$ sobre raps. En general, los cultivos siguieron la tendencia avena $>$ raps $>$ lupino.

En el Inceptisol no se encontraron diferencias entre los dos cultivos no micorrizables mientras la avena, un cereal bastante microtrófico, incrementó las esporas en alrededor de $640 \%$ en relación con los cultivos no hospederos.

Los resultados obtenidos para las esporas HMA concuerdan con lo informado por Castillo (2005) en un ensayo en campo con rotación corta Triticum aestivum - L. albus, donde la leguminosa no hospedera produjo un descenso drástico de las poblaciones fúngicas. Por el contrario, Millaleo et al. (2006) en una rotación de cultivos realizada en un Ultisol adicionado con distintas dosis 


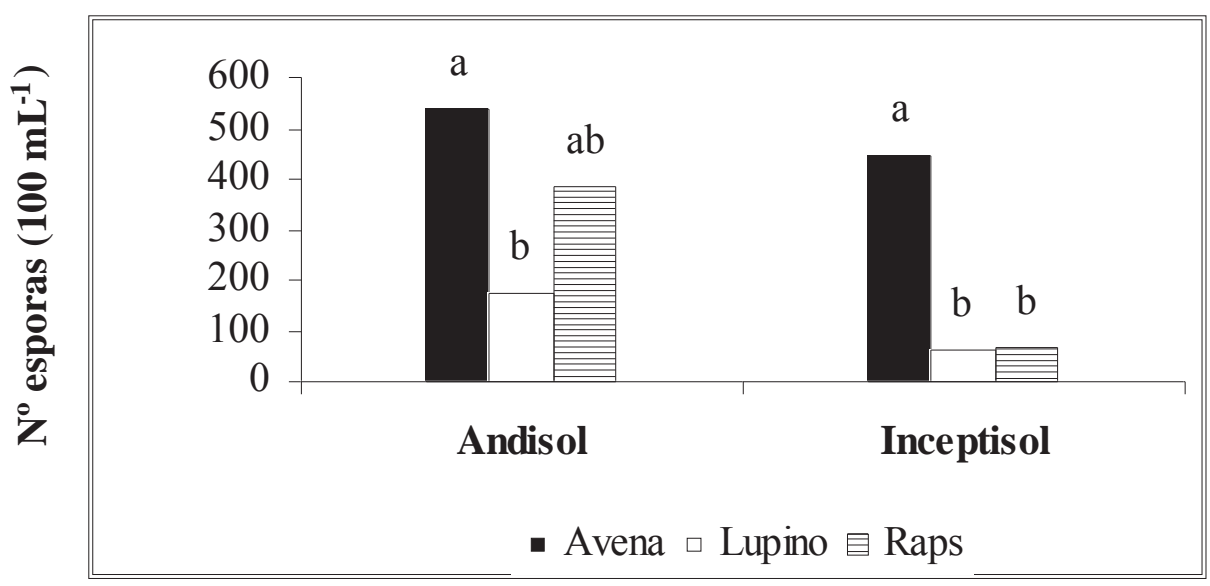

Figura 1. Número de esporas de HMA aisladas desde un Andisol e Inceptisol de la Región de La Araucanía, Chile. En cada suelo barras con la misma letra no difieren estadísticamente, test de Tukey $(\alpha=0,05)$.

Figure 1. AMF spore number isolated from an Andisol and Inceptisol in the Región de La Araucanía, Chile. In each soil bars with the same letter did not differ significantly, Tukey test $(\alpha=0.05)$.

de compost, en condiciones de invernadero y utilizando como hospederos Phaseolus vulgaris, T. aestivum y Lolium perenneTrifolium pratense informaron para todos los cultivos, un número de esporas muy superior a los obtenidos en este estudio. La diferencia se podría explicar por la utilización de cultivos micotróficos y por el potencial fúngico del suelo Lumaco, que probablemente fue superior al utilizado en este trabajo. La variabilidad en el número de esporas entre cultivos podría deberse a que las asociaciones micorrícicas que se consideraban no específicas (cualquier hongo simbionte puede colonizar a cualquier planta receptiva) según van der Heijden et al. (1998) existirían ciertas preferencias o mejor compatibilidad entre determinados hongos y plantas, lo que en este estudio se demostró en el suelo Temuco cultivado con avena. Por otra parte, Mathimaran et al. (2005) encontraron que la formación de micorrizas se inhibe en suelos arcillosos, debido a la dificultad de las raíces para penetrarlo así como también, a la escasa aireación, lo que podría explicar el bajo número de esporas encontradas en el suelo Lumaco.

El micelio fúngico (Figura 2) presentó diferencias significativas en el suelo Temuco, contabilizándose una cantidad mayor en el cultivo no micotrófico raps, respecto de lupino. En el caso de avena, cultivo que es altamente micorrizable, no hubo diferencias con los cultivos no hospederos. Por el contrario, en el suelo Lumaco en comparación con el Andisol, la densidad de hifas fue mayor con avena y lupino mientras que, en raps se encontró menor longitud; sin embargo, no se encontraron diferencias significativas entre los cultivos. Estos resultados concuerdan con lo informado por Millaleo et al. (2006), quienes no encontraron diferencias en el 


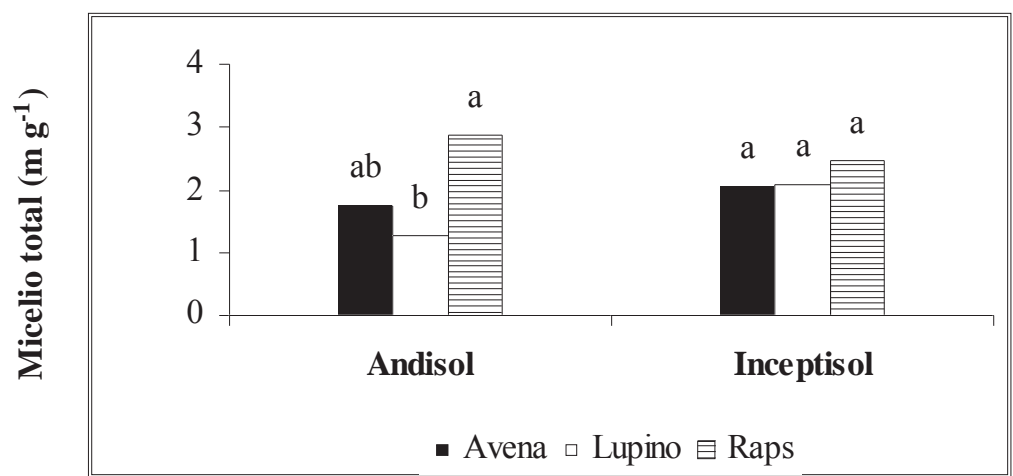

Figura 2. Micelio total HMA aislado desde un Andisol e Inceptisol de la Región de La Araucanía, Chile. En cada suelo barras con la misma letra no difieren estadísticamente, test de Tukey $(\alpha=0,05)$.

Figure 2. Total AMF mycelium isolated from an Andisol and Inceptisol in the Región de La Araucanía, Chile. In each soil bars with the same letter did not differ significantly, Tukey test $(\alpha=0.05)$.

micelio total, en una rotación orgánica con trigo y pradera mientras que, cultivado con frejol aumentaron los propágulos. La colonización HMA en las raíces sólo se cuantificó en avena, porque la familia Brassicaceae y el género Lupinus no forman micorrizas. En el Andisol Temuco, la colonización fue levemente superior (35\%) al Inceptisol (31\%).

La colonización HMA encontrada en avena crecida en el Andisol y en el Inceptisol en condiciones de invernadero, resultó baja en comparación a valores encontrados en esta misma especie, pero en condiciones de campo, donde puede variar entre $45 \%$ en sistemas de labranza convencional a $55 \%$ con cero labranza (Castillo et al., 2006). Las diferencias podrían explicarse por las condiciones muy diferentes entre un cultivo crecido en invernadero con los de campo, donde probablemente el potencial fúngico se encuentra exacerbado, por la presencia de una gran variedad de malezas que son hospederos, que generalmente acompañan a un cultivo.

En relación a los parámetros ecológicos, en ambos suelos, resulta interesante destacar la riqueza fúngica ( $R$ ) que fue significativamente superior en avena, cultivo micotrófico, que en lupino y raps, estos dos últimos, no hospederos (Cuadro 2). En el Andisol, se encontraron 33 morfotipos HMA mientras que en lupino y raps no hubo diferencias con un R igual a 23, mostrando la avena un incremento en $43 \%$ sobre los otros cultivos. En Inceptisol, la avena nuevamente tuvo mayor R (22), acentuándose las diferencias con incrementos de $69 \%$ en relación al lupino y de $100 \%$ sobre raps. Estos resultados en el Inceptisol, para los tres cultivos, son mayores a los obtenidos por Castillo et al. (2004) en un Ultisol en campo sembrado con lupino, después de varios años de pradera natural, donde $\mathrm{R}$ fluctuó entre $8 \mathrm{y}$ 9 y los informados por Serralde y Ramírez (2004) quienes señalaron un promedio de 24 morfotipos en suelos ácidos cultivados con maíz.

En el Andisol, el índice de ShannonWiener presentó diferencias significativas entre raps y lupino, siendo la diversidad mayor con lupino (Cuadro 2), mientras en el Inceptisol para esta variable no se 
Cuadro 2. Índices de diversidad de HMA en un Andisol e Inceptisol de la Región de La Araucanía, Chile. En cada columna y para cada suelo, valores con letras distintas difieren significativamente, según t-Student $(\alpha=0,05)$.

Table 2. Diversity index of AMF in an Andisol and Inceptisol in the Región de La Araucanía, Chile. In the column and for each soil, values with different letter differ significantly, according t-Student $(\alpha=0.05)$.

\begin{tabular}{cccccc}
\hline Suelo & Cultivo & Riqueza & $\begin{array}{c}\text { Shannon- } \\
\text { Wiener }\end{array}$ & Simpson & Equidad \\
\hline \multirow{3}{*}{ Andisol } & Avena & $33 \mathrm{a}$ & $2,43 \mathrm{~b}$ & 0,18 & 0,69 \\
& Lupino & $23 \mathrm{~b}$ & $2,62 \mathrm{a}$ & 0,09 & 0,84 \\
& Raps & $23 \mathrm{~b}$ & $2,43 \mathrm{ab}$ & 0,11 & 0,79 \\
& Avena & $22 \mathrm{a}$ & $2,27 \mathrm{a}$ & 0,15 & 0,73 \\
\multirow{3}{*}{ Inceptisol } & Lupino & $13 \mathrm{~b}$ & $2,39 \mathrm{a}$ & 0,09 & 0,93 \\
& Raps & $11 \mathrm{~b}$ & $1,95 \mathrm{a}$ & 0,18 & 0,81 \\
\hline
\end{tabular}

encontraron diferencias significativas en el análisis de varianza $(\alpha=0,05)$. Resulta interesante destacar que en el Andisol, el lupino presentó la mayor diversidad junto con una relativamente alta riqueza (23) en comparación con la avena (33), lo que podría señalar que la distribución de las especies dentro de la población fúngica fue bastante homogénea, siendo lo anterior corroborado por un bajo índice de dominancia.

Bajo las condiciones evaluadas se observó una diversidad alta de acuerdo a los índices ecológicos calculados para los tres cultivos, si se consideran los resultados reportados para poblaciones MA en diversos agroecosistemas. Así, Collins (1991) encontró valores de $\mathrm{H}^{`}$ que variaron entre 0,42 y 1,59 para cultivos de maíz y soja. Por otra parte, Castillo et al. (2004) en un estudio en campo realizado en la Región de La Araucanía, reportó una media de $\mathrm{H}^{`}$ igual a 1,7. Además, el H`obtenido en lupino resulta mayor al informado por Blaszkowski (2003) en suelos cultivados de Polonia con un valor de H`que fluctuó entre 1,8 y 2,2 mientras que, en un estudio realizado por Franke-Snyder et al. (2001) el valor H' resultó ser igual a 1,76 . Con los resultados obtenidos para el análisis de la población global se observa que en el Inceptisol hubo un efecto de los cultivos no hospederos sobre las poblaciones HMA, a nivel de género.

La Figura 3 muestra los contenidos de materia seca de los tres cultivos. En el lupino, la mayor masa se obtuvo en el Andisol, con un incremento de $19 \%$ sobre el que tuvo en el Inceptisol. Esta diferencia podría relacionarse con la gran actividad fosfatásica del suelo cultivado con lupino (Figura 4) ya que, la enzima al liberar P desde el pool orgánico del suelo mejora la nutrición fosforada, permitiendo un mejor desarrollo de la leguminosa. Los otros dos cultivos tuvieron mayor masa en el Inceptisol; la avena incrementó $31 \%$ y el raps $27 \%$ en relación al peso mostrado en el Andisol. 


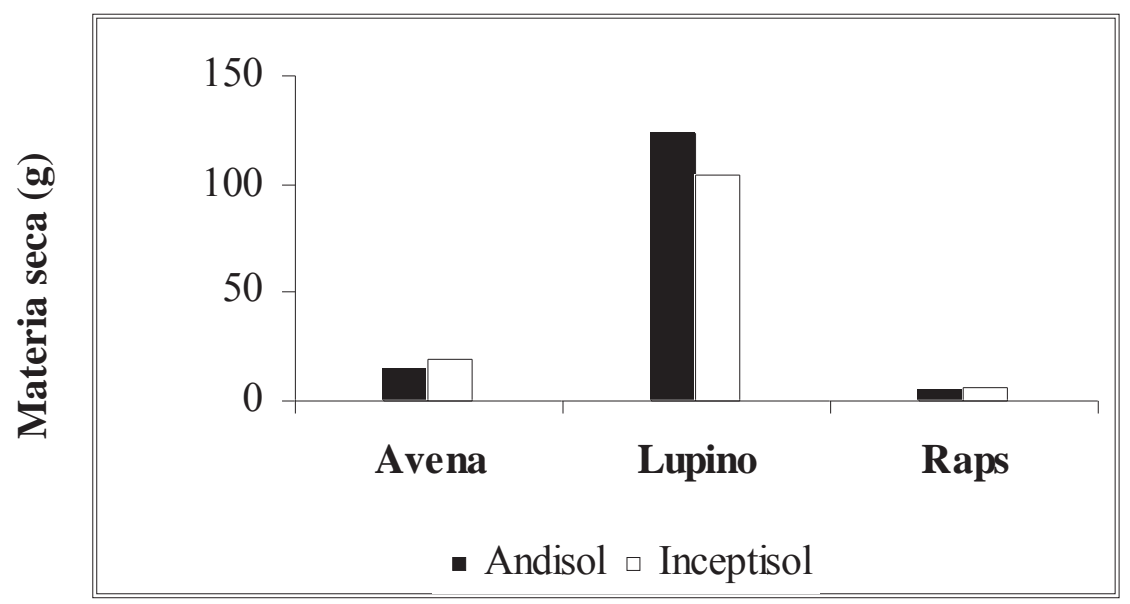

Figura 3. Materia seca en tres cultivos crecidos en un Andisol e Inceptisol de la Región de La Araucanía, Chile. No se presentaron diferencias significativas entre los dos tipos de suelo dentro de una especie.

Figure 3. Dry matter in three grown cultures in an Andisol and Inceptisol in the Región de La Araucanía, Chile. There were not significant differences between two types of soil into specie.

La actividad de cualquier enzima en el suelo está relacionada ya sea, con factores bióticos como abióticos y la fase acuosa pero, su localización cambia con el tiempo, pudiendo servir como medida indirecta de la biomasa microbiana, como un indicador de la potencialidad del suelo para degradar la MO o también, como guía de la productividad de un suelo. En este estudio sobre enzimas se evaluó solamente la fosfatasa ácida, que es la forma predominante en suelos ácidos, donde los resultados encontrados en el Andisol con lupino resultaron ser bastante elevados. En ambos suelos, como se muestra en la Figura 4, los contenidos de enzima presentaron diferencias significativas $(\alpha=0,05)$ entre lupino con avena y raps. Así, en el Andisol, el lupino incrementó notablemente la actividad en $526 \%$ sobre avena y en $419 \%$ respecto al raps mientras que, en Inceptisol los incrementos fueron menores, pero siempre significativos de $250 \%$ sobre avena y $159 \%$ en relación al raps. Entre el hospedero avena y el no hospedero raps no se aprecian mayores diferencias en las cantidades de enzima liberadas al suelo, siendo superiores en el Andisol. Fluctuaciones en el contenido de fosfatasas en suelos con distintos cultivos, han sido informadas por Tadano (1993) quien encontró que la habilidad que presentan las raíces para secretar fosfatasas difiere entre especies de plantas e inclusive entre organismos de una misma especie. Por otra parte, Portilla et al. (1998) encontraron que la actividad fosfatásica varía inter e intraespecíficamente siendo influenciada por la madurez fisiológica de la planta y presentando respuestas diferentes según las variadas formas de $\mathrm{P}$ orgánico e inorgánico del suelo. 


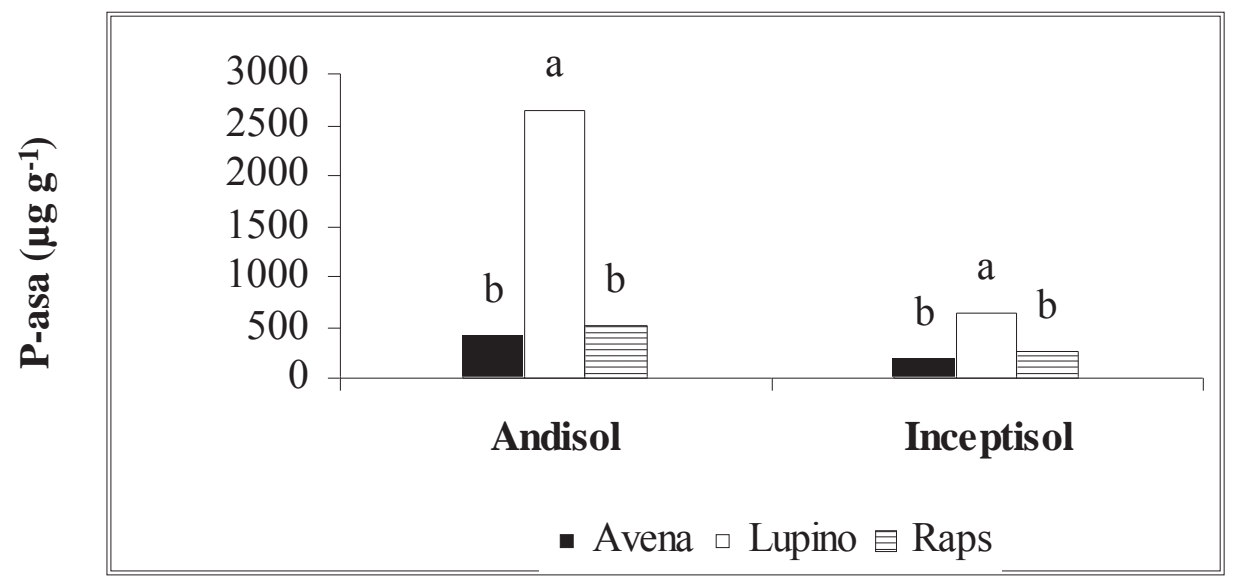

Figura 4. Actividad fosfatásica en un Andisol e Inceptisol de la Región de La Araucanía, Chile. En cada suelo barras con la misma letra no difieren estadísticamente, test de Tukey $(\alpha=0,05)$.

Figure 4. Phosphatase activity in an Andisol and Inceptisol in the Región de La Araucanía, Chile. In each soil bars with the same letter did not differ significantly, Tukey test $(\alpha=0.05)$.

En estudios realizados por Borie et al. (1998) informaron que la actividad de la enzima fue mayor en plantas crecidas en un suelo estéril y que en el ciclado del $\mathrm{P}$ la micorrización sería un mecanismo complementario de la actividad fosfatásica. Además, Castillo (2005) en un ensayo en campo, encontró que en una rotación cereal - leguminosa, cuando se incluía lupino, se producía un descenso drástico en la densidad de esporas, acompañado de un aumento en la actividad fosfatásica, demostrando que ambos mecanismos actuarían sinérgicamente en el ciclado y aprovechamiento del P siendo estos estudios nuevamente corroborados en este trabajo en el Inceptisol con lupino ya que, al aumentar la actividad enzimática se produjo una disminución en la concentración de esporas.

En relación a la colonización HMA, Portilla et al. (1998) informaron que una disminución en el porcentaje de colonización se compensa, por otros mecanismos, como un aumento en la actividad fosfatásica. Lo anterior se observó en el cultivo de avena; así, con una colonización HMA relativamente alta que fluctuó entre $31 \%$ y $35 \%$, el contenido de la enzima descendió a niveles bajos entre $0,18 \mathrm{mg} \mathrm{g}^{-1}$ y $0,42 \mathrm{mg}$ $\mathrm{g}^{-1}$. Al respecto, Rubio et al. (1990) encontraron que la colonización HMA correlacionó negativamente con la actividad fosfatásica, utilizando la planta estos dos mecanismos en forma complementaria para la adquisición de $\mathrm{P}$ por lo que, en suelos derivados de cenizas volcánicas, cultivados con A. sativa y L. albus, con ayuda de la simbiosis micorrícica o actividad fosfatásica, se favorece la disponibilidad del nutriente. Al disminuir los propágulos HMA en la rizósfera de lupino, un cultivo no micotrófico, se produce un aumento en la exudación de la enzima desde las raíces proteoídeas de la leguminosa, lo que eleva el contenido de fosfatasas en el suelo.

Respecto a los parámetros edáficos evaluados, en Andisol, el P disponible presentó diferencias entre los tres cultivos, destacando el suelo con raps, con una significativa disponibilidad del nutriente, 
incrementando en $140 \%$ su contenido respecto al lupino y $58 \%$ sobre avena (Figura 5). En Inceptisol, estas diferencias fueron aún más notorias que en el Andisol; así, raps aumentó en $1029 \%$ sobre lupino y $190 \%$ en relación al cultivo de avena. Al respecto, Portilla et al. (1998) informan que la simbiosis entre plantas hospederas con hongos micorrícicos, capta más $\mathrm{P}$ que cultivos no micorrizados. Por otra parte, Friberg (2001) informó una relación inversa entre la disponibilidad del nutriente con la colonización HMA en las raíces; sin embargo, en el Andisol, se observó una relación directa entre el porcentaje HMA y el contenido de P (Cuadro 2, Figura 5).

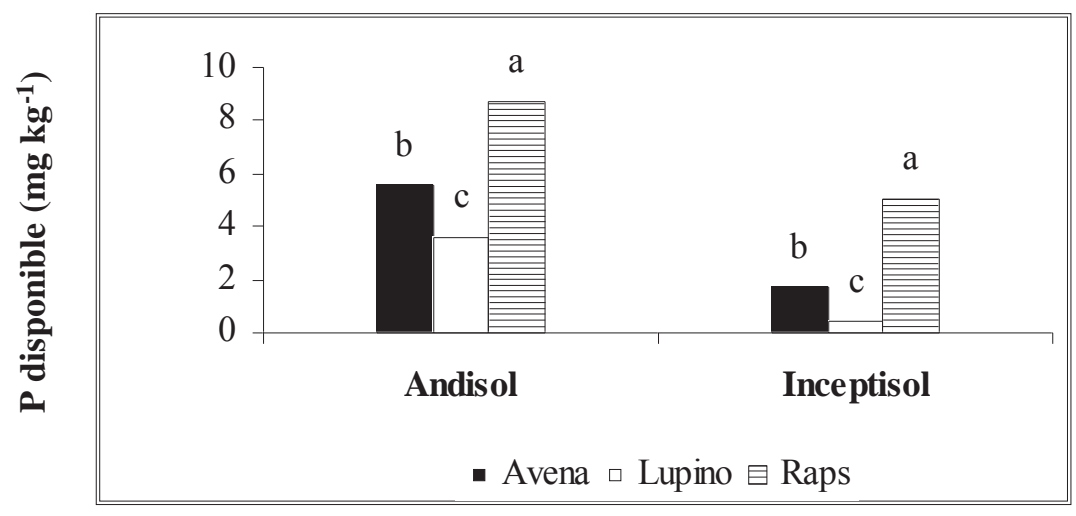

Figura 5. Fósforo disponible en un Andisol e Inceptisol de la Región de La Araucanía, Chile. En cada suelo barras con la misma letra no difieren estadísticamente, test de Tukey $(\alpha=0,05)$.

Figure 5. Available phosphorus in an Andisol and Inceptisol in the Región de La Araucanía, Chile. In each soil bars with the same letter did not differ significantly, Tukey test $(\alpha=0.05)$.

Otro parámetro edáfico que se determinóen el estudio fue el pH (Cuadro 3), que en Andisol no presentó diferencias entre cultivos. Este tipo de suelos tiene como principal factor limitante para el desarrollo de las plantas, la acidez, que afecta la disponibilidad de nutrientes esenciales principalmente, P y N (Montaño et al., 2001). El Inceptisol con avena presentó la menor acidez, con incrementos en 0,35 unidades de $\mathrm{pH}$ sobre lupino, cultivo que en ambos suelos presentó la mayor acidez debido, probablemente a la exudación de ácidos quelantes por sus raíces proteoídeas. Al respecto, Friberg (2001) encontró que el pH influye sobre algunas especies HMA en su capacidad colonizadora de raíces.

En el Andisol, se encontraron diferencias en el contenido de $\mathrm{P}$ total (Cuadro 3) entre la avena con los otros cultivos no hospederos, siendo estos últimos, los que tuvieron los mayores contenidos del nutriente y al comparar los valores con el suelo inicial (Cuadro 1) se observa que prácticamente se mantuvieron constantes. El Inceptisol cultivado con raps presentó la mayor cantidad de $\mathrm{P}$ con diferencias significativas en relación con avena y lupino. 
Cuadro 3. Caracterización del Andisol e Inceptisol de la Región de La Araucanía, Chile. Para un mismo suelo y en una misma fila, valores con letras distintas presentan diferencias estadísticamente significativas, según test Tukey $(\alpha=0,05)$.

Table 3. Characterization of the Andisol and Inceptisol in the Región de La Araucanía, Chile. For each soil in the same file values with different letters are different, according Tukey test ( $\alpha=0.05)$.

\begin{tabular}{lccccccc}
\hline Variable & \multicolumn{3}{c}{ Andisol } & & \multicolumn{3}{c}{ Ultisol } \\
\cline { 2 - 4 } \cline { 7 - 8 } & Avena & Lupino & Raps & & Avena & Lupino & Raps \\
\hline $\mathrm{pH}$ & $5,99 \mathrm{a}$ & $5,89 \mathrm{a}$ & $6,02 \mathrm{a}$ & & $5,63 \mathrm{a}$ & $5,28 \mathrm{c}$ & $5,49 \mathrm{~b}$ \\
Humedad (\%) & $26 \mathrm{c}$ & $55 \mathrm{a}$ & $42 \mathrm{~b}$ & & $4 \mathrm{c}$ & $14 \mathrm{~b}$ & $19 \mathrm{a}$ \\
$\mathrm{D}_{\mathrm{ap}}{ }^{1}\left(\mathrm{~g} \mathrm{~mL}^{-1}\right)$ & $0,53 \mathrm{a}$ & $0,35 \mathrm{~b}$ & $0,46 \mathrm{a}$ & & $1,16 \mathrm{a}$ & $0,93 \mathrm{~b}$ & $0,84 \mathrm{c}$ \\
$\mathrm{P}$ total $\left(\mathrm{mg} \mathrm{kg}^{-1}\right)$ & $2936 \mathrm{~b}$ & $3148 \mathrm{a}$ & $3194 \mathrm{a}$ & & $1004 \mathrm{~b}$ & $1065 \mathrm{~b}$ & $1141 \mathrm{a}$ \\
$\mathrm{Na}\left(\mathrm{cmol}_{+} \mathrm{kg}^{-1}\right)$ & $0,42 \mathrm{a}$ & $0,48 \mathrm{a}$ & $0,50 \mathrm{a}$ & & $0,38 \mathrm{a}$ & $0,41 \mathrm{a}$ & $0,21 \mathrm{a}$ \\
$\mathrm{Fe}\left(\mathrm{mg} \mathrm{kg}^{-1}\right)$ & $14,94 \mathrm{a}$ & $22,96 \mathrm{a}$ & $17,02 \mathrm{a}$ & & $16,54 \mathrm{a}$ & $17,20 \mathrm{a}$ & $12,51 \mathrm{~b}$ \\
$\mathrm{Al}\left(\mathrm{mg} \mathrm{kg}^{-1}\right)$ & $17,90 \mathrm{a}$ & $16,82 \mathrm{a}$ & $16,13 \mathrm{a}$ & $33,50 \mathrm{c}$ & $85,05 \mathrm{a}$ & $56,93 \mathrm{~b}$ \\
\hline
\end{tabular}

${ }^{\mathrm{I} D e n s i d a d ~ a p a r e n t e}$

Por su parte, Borie et al. (1989) informaron que los suelos de la Región de La Araucanía se caracterizan por tener una baja disponibilidad de nutrientes, con elevada cantidad en $\mathrm{P}$ total y en suelos agrícolas todas las formas de $\mathrm{P}$ pueden encontrarse potencialmente disponibles para las plantas, dependiendo entre otros factores del ambiente suelo-raíz.

En Andisol, las concentraciones de K no presentaron diferencias, mientras en el Inceptisol la avena tuvo un contenido 175 $\%$ superior al suelo con raps (Figura 6). El otro catión intercambiable, Ca, en el
Inceptisol presentó significativamente mayor contenido con avena que con los otros cultivos, mientras en el Andisol no se encontraron diferencias entre tratamientos, pero siendo todos los contenidos mayores que en el Inceptisol (Figura 6). Mg, otro macronutriente de los que se analizaron por EAA tuvo mayor concentración en el Andisol (raps $=2,01 \mathrm{cmol}_{+} \mathrm{kg}^{-1}$; lupino $=$ $1,77 \mathrm{cmol}_{+} \mathrm{kg}^{-1}$; avena $=1,62 \mathrm{cmol}_{+} \mathrm{kg}^{-1}$ ) que en el Inceptisol (raps $=0,68 \mathrm{cmol}_{+}$ $\mathrm{kg}^{-1}$; lupino $=0,96 \mathrm{cmol}_{+} \mathrm{kg}^{-1}$; avena $=1,03$ $\mathrm{cmol}_{+} \mathrm{kg}^{-1}$ ), pero sin diferencias entre los cultivos. 


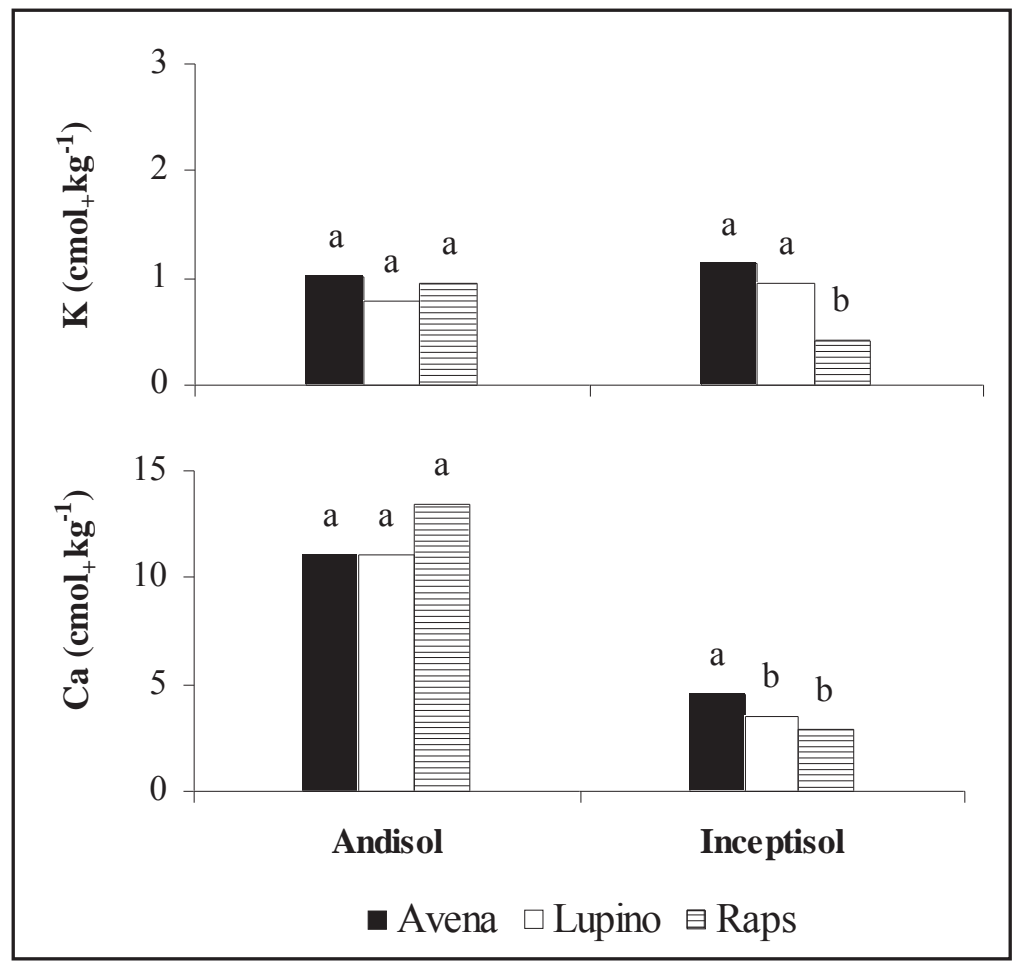

Figura 6. Concentración de macronutrientes en un Andisol e Inceptisol de la Región de La Araucanía, Chile. En cada suelo barras con la misma letra no difieren estadísticamente, test de Tukey $(\alpha=0,05)$.

Figure 6. Macronutrients concentration of in an Andisol and Inceptisol in the Región de La Araucanía, Chile. In each soil bars with the same letter did not differ significantly, Tukey test $(\alpha=0.05)$.

En relación con el análisis de micronutrientes la concentración de $\mathrm{Cu}$, en los suelos fue semejante en los tres cultivos, encontrándose mayores concentraciones en el Inceptisol (Figura 7). En el Andisol, el Zn presentó diferencias $(\alpha=0,05)$ entre los dos cultivos no micorrizables, raps con lupino, teniendo el raps un incremento de $62 \%$ en relación a lupino. En Inceptisol con avena, se incrementó notablemente el contenido del nutriente $(321 \%)$ en comparación con los cultivos no hospederos.

En general, de acuerdo a los resultados presentados en la Figura 7, se puede observar que el Inceptisol con cultivo hospedero presentó contenidos mayores de micronutrientes que el Andisol, suelo donde las diferencias en las concentraciones de nutrientes fueron mínimas para los tres cultivos. Al respecto, Guadarrama et al. (2004) informan que los HMA aumentan la capacidad de adquisición y asimilación de recursos por parte de la planta hospedera ya que, en comparación con las raíces, las hifas externas de estos hongos poseen mayor habilidad para explorar el suelo, mejorando así, la captación de nutrientes. Además, según Clark y Zeto (2000) la adquisición de $\mathrm{K}, \mathrm{Ca}, \mathrm{Mg}, \mathrm{Zn}$ y $\mathrm{Cu}$ se incrementa por la inoculación con HMA en suelos ácidos. 


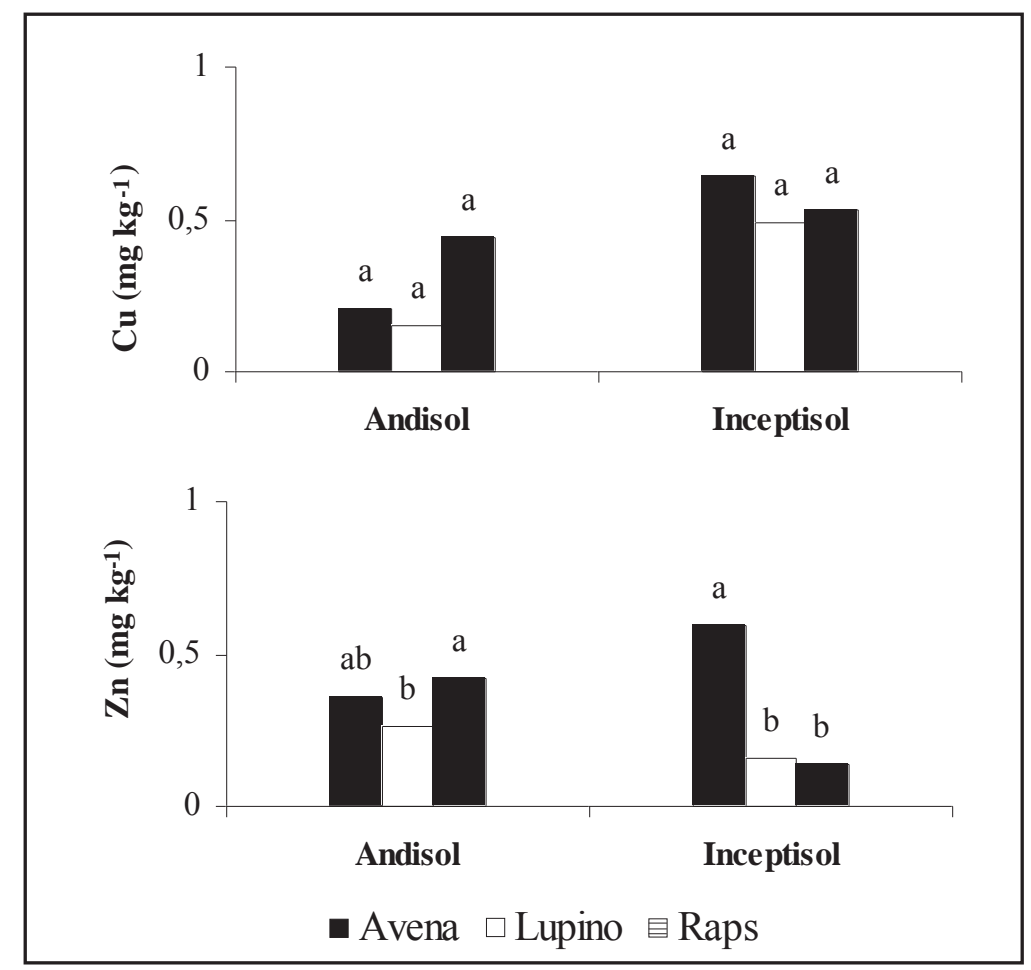

Figura 7. Concentraciones de micronutrientes en un Andisol e Inceptisol de la Región de La Araucanía, Chile. En cada suelo barras con la misma letra no difieren estadísticamente, test de Tukey $(\alpha=0,05)$.

Figure 7. Micronutrients concentration of in an Andisol and Inceptisol in the Región de La Araucanía, Chile. In each soil bars with the same letter did not differ significantly, Tukey test $(\alpha=0.05)$.

Finalmente, el contenido de $\mathrm{Mn}$ en ambos suelos, no fue diferente, pero mientras en el Andisol prácticamente no se pudo detectar por el método instrumental utilizado, en el Inceptisol se encontraron elevados contenidos que fluctuaron entre $33 \mathrm{mg} \mathrm{kg}^{-1}$ a $38 \mathrm{mg} \mathrm{kg}^{-1}$

En el Cuadro 4, al correlacionar en el Andisol el número de esporas con la actividad fosfatásica se obtuvo una relación de tipo positiva; por el contrario, en el Inceptisol, las dos variables se relacionaron negativamente. Sin embargo, en este último suelo se alcanzó una interacción de tipo positiva entre el número de esporas con el contenido de $\mathrm{MO}$, relación más estrecha que la encontrada por Castillo (2005) para una rotación en campo trigo-lupino, con cero labranza $\left(r^{2}=0,31\right)$ y en labranza convencional $\left(r^{2}=0,36\right)$. 
Cuadro 4. Coeficientes de correlación entre número de esporas y los parámetros de los suelos de la Región de La Araucanía.

Table 4. Correlations coefficient between spore number and soils parameters of the Región de La Araucanía.

\begin{tabular}{lcc}
\hline Correlación $^{\mathbf{1}}$ & Andisol & Inceptisol \\
\hline Esporas v/s P-asa & 0,77 & $-0,55$ \\
Esporas v/s MO (\%) & $-0,34$ & 0,91 \\
Esporas v/s humedad (\%) & $-0,80$ & $-0,86$ \\
Esporas v/s pH & 0,27 & 0,48 \\
Esporas v/s P disponible & 0,33 & $-0,11$ \\
\hline
\end{tabular}

${ }^{1} \mathrm{n}=9$ para cada variable.

\section{CONCLUSIONES}

El cultivo no micotrófico Lupinus albus afectó significativamente la densidad de esporas HMA en el Andisol, no así Brassica napus, el otro cultivo no micotrófico, que no presentó diferencias significativas con Avena sativa, cultivo altamente micorrizable. En el Inceptisol, ambos cultivos no micotróficos disminuyeron considerablemente el número de esporas en relación a la Avena sativa mientras que, el porcentaje de colonización HMA en las raíces de avena fue menor en el Inceptisol que en el Andisol, encontrándose en éste último la mayor cantidad de micelio total con raps. En avena ya sea, en el Andisol como Inceptisol, se encontró la mayor riqueza de especies.
La actividad fosfatásica, en ambos suelos, presentó diferencias significativas con el cultivo no micotrófico Lupinus albus en comparación a los otros dos cultivos siendo mayor en el Andisol que en el Inceptisol siguiendo ambos la tendencia avena $<$ raps $<$ lupino.

Este estudio sobre efecto de cultivos hospederos y no hospederos sobre los propágulos fúngicos en suelos típicos de la Región de La Araucanía, deja abierta la interrogante de qué sucedería con los propágulos al rotar los tres cultivos con un cereal micorrizable como Triticum aestivum. 


\section{BIBLIOGRAFÍA}

ALLEN, B.E., ALLEN, M.F., (1990). The mediation of competition by mycorrhizae in successional and patchy environments, in: J.B. Grace, D. Tilman, (eds). Perspectives on Plant Competition. Academic Press, London. $484 \mathrm{p}$.

AUGÉ, R. M., 2004. Arbuscular mycorrhizae and soil/plant water relations. J. Soil Sci. 84: 373-381.

BELLGARD, S.E., 1993. The topsoil as the major store of propagules of vesiculararbuscular fungi in southeast Australian sandstone soils. Mycorrhiza 3: 19-24.

BLASZKOWSKI, J., 2003. Arbuscular mycorrhizal fungi (Glomeromycota), Endogone and Complexipes species deposited in the Departament of Plant Pathology, University of Agriculture in Szczecin, Poland. Available at http://www.agro.ar.szczecin.pl/ jblas zkowski/index.html Accessed 26 july 2007.

BORIE, F., RUBIO, R., 1999. Effects of arbuscular mycorrhizae and liming on growth and mineral acquisition of aluminum-tolerant and aluminumsensitive barley cultivars. J. Plant Nutr. 22: 121-137.

BORIE, F., ZUNINO, H., MARTÍNEZ, L., 1989. Macromolecule P-associations and inositol phosphates in some Chilean volcanic soils of temperate regions. Comm. Soil Sci. Plant Anal. 20: 18811894.

BORIE, F., RUBIO, R., SCHALCHLI, C., 1998. Micorrizas arbusculares y actividad fosfatásica de diez cultivares de trigo. Agric. Téc. 58(1): 47-55.

CASTILLO, C.G., 2005. Biodiversidad y efectividad de hongos micorrícicos arbusculares en ecosistemas agroforestales del Centro Sur de Chile. Tesis Doctoral, Universidad de La Frontera. $124 \mathrm{p}$.
CASTILLO, C.G., RUBIO, R., CONTRERAS, A., BORIE, F., 2004. Hongos micorrizógenos arbusculares en un Ultisol de la IX Región fertilizado orgánicamente. R. C. Suelo Nutr. Veg. 4(2): 39-47.

CASTILLO, C.G., RUBIO, R., ROUANET, J., BORIE, F., 2006. Early effects of tillage and crop rotation on arbuscular mycorrhizal fungal propagules in an Ultisol. Biol. Fert. Soils 43: 83-92.

CLARK, R., ZETO, S., 2000. Mineral acquisition by mycorrhizal plants. J. Plant Nutr. 23: 867-902

COLLINS, N., PFLEGER, E., CROVKSTON, R., SIMMONS, S., COIPELAND, P., 1991. Vesiculararbuscular mycorrhizal respond to corn and soybean cropping history. New Phytol. 117: 657-663.

DICK, W.A., TABATABAI, M.A., 1977. An alkaline oxidation method for the determination of total phosphorus in soils. J. Soil Sci. Soc. Am. 41: 511514.

EISSENSTAT, D.M., NEWMAN, E.I., 1990. Seedling establishment near large plants: effects of vesicular-arbuscular mycorrhizas on intensity of plant competition. Funct. Ecol. 4: 95-99.

FRANKE-SNYDER, M., DOUDS, D., GALVEZ, L., PHILLIPS, J., WAGONER, P., DRINKWATER, L., MORTON, J., 2001. Diversity of communities of arbuscular mycorrhizal (AM) fungi present in conventional versus low-input agricultural sites in eastern Pennsylvania, USA. Appl. Soil Ecol. 16: 35-48.

FRIBERG, S., 2001. Distribution and diversity of arbuscular mycorrhizal fungi in traditional agriculture on the Niger inland delta, Mali, West Africa. CBM:s Skriftserie 3: 53-80. 
GIOVANETTI, M., MOSSE, B., 1980. An evaluation of techniques for measuring vesicular-arbuscular mycorrhizal infection in roots. New Phytol. 84: 489-500.

GUADARRAMA, P., SÁNCHEZGALLÉN, I., ÁLVAREZ-SÁNCHEZ, J., RAMOS-ZAPATA, J., 2004. Hongos y plantas, beneficios a diferentes escalas en micorrizas arbusculares. Ciencias 73:38-45.

HEREDIA, A.G., (2003). Los hongos microscópicos en la descomposición de las hojas, en: J. Álvarez-Sánchez, E. Naranjo-García (eds). Ecología del Suelo en la Selva Tropical Húmeda de México. Instituto de Ecología AC, Instituto de Biología y Facultad de Ciencias, UNAM. Xalapa. 316 p.

JASPER, D.A., ABBOTT, L.K., ROBSON, A.D., 1993. The survival of infective hyphae of vesicular-arbuscular mycorrhizal fungi in dry soil: an interaction with sporulation. New Phytol. 124: 473-479.

LINDERMAN, R.G., DAVIS, E.A., 2004. Varied response of marigold (Tagetes spp.) genotypes to inoculation with different arbuscular mycorrhizal fungi. Sci. Hort. 99: 67-78.

MAGURRAN, A.E., (1988). Ecological diversity and its measurement. Princeton University Press, New Jersey, 179 p.

MATHIMARAN, N., RUH, R. VULLIOUD, P., FROSSARD, E., JANSA, J., 2005. Glomus intraradices dominates arbuscular mycorrhizal communities in a heavy textured agricultural soil. Mycorrhiza 16: 61-66.

McGONIGLE, T.P., FITTER, A.H., 1990. Ecological specificity of vesiculararbuscular mycorrhizal associations. Mycol. Res. 94: 120-122.

MENDOZA, J., BORIE, F., 1998. The effect of Glomus etunicatum inoculation on aluminum, phosphorus, calcium and magnesium uptake in two barley genotypes with different aluminum tolerance. Commun. Soil Sci. Plant Anal. 9: 681-695.
MillaleO, R., MONTECINOS, C., RUBIO, R., CONTRERAS, A., BORIE, F., 2006. Efecto de la adición de compost sobre propágulos micorrícicos arbusculares en un suelo volcánico del centro sur de Chile. R.C. Suelo Nutr. Veg. 6(3): 26-39.

MONTAÑO, N., QUIROZ, V., CRUZFLORES, G., 2001. Colonización micorrícica arbuscular y fertilización mineral de genotipos de maíz y trigo cultivados en un Andisol. Facultad de estudios superiores Zaragoza, Universidad Nacional Autónoma de México. México.

MORENO, C., (2001). Métodos para medir la biodiversidad. M\&T-Manuales y Tesis SEA. Vol. 1. Zaragoza. 84 p. OLSEN, S.R., SOMMERS, L.E., (1982): Phosphorus, in A.L. Page et al. (eds) Methods of Soil Analysis. Part. 2. 2nd ed. Agron. Monogr. 9. ASA and SSSA, Madison, Wisconsin, USA. p. 403-430. PHILLIPS, J.M., HAYMAN, D.S., 1970. Improved procedures for clearing roots and staining parasitic and vesiculararbuscular mycorrhizal fungi for rapid assessment of infection. Trans. Brit. Mycol. Soc. 55: 158-161.

PORTILlA, I., MOLINA, E., CRUZ, G., ORTIZ, I., MANSKE, G.G.B., 1998. Colonización micorrízica arbuscular, actividad fosfatásica y longitud radical como respuesta a estrés de fósforo en trigo y triticale cultivados en un Andisol. TERRA 16 (1): 55-61.

REEVES, F.B., 1985. Survival of VA mycorrhizal fungi-interactions of secondary succession, mycorrhizal dependency in plants, and resource competition. In: Molina, R. (ed.). Proc. North Am. Conf. on Mycorrhizae, 6th, Bend, OR. 25-29 June 1984. Forest Res. Lab., Oregon State Univ., Corvallis, p. 110-113.

RUBIO, R., MORAGA, E., BORIE, F., 1990. Acid phosphatase activity and vesicular-arbuscular infection associated with roots of four wheat cultivars. J. Plant Nutr. 13: 585-598. 
RUBIO, R., BORIE, F., SCHALCHLI, C., CASTILlO, C., AZCÓN, R., 2003. Occurrence and effect of arbuscular mycorrhizal propagules in wheat as affected by the source and amount of phosphorus fertilizer and fungal inoculation. Appl. Soil Ecol. 23: 245255.

SADZAWKA, A., GREZ, R., MORA, M.L., SAAVEDRA, N., CARRASCO, M.A., ROJAS, C., 2000. Métodos de análisis recomendados para los suelos chilenos. Sociedad Chilena de la Ciencia del Suelo, Santiago, Chile, 62 p.

SERRALDE, A.M., RAMÍREZ, M.M., 2004. Análisis de poblaciones de micorrizas en maíz (Zea mayz) cultivado en suelos ácidos bajo diferentes tratamientos agronómicos. Revista Corpoica 5(1): 31-40.

SIEVERDING, E., 1991. Vesiculararbuscular mycorrhiza management in tropical agrosystems in Deutsche Gesellschaft für Technische Zusammenarbeiit (GTZ), GmbH, Eschborn, Germany, $371 \mathrm{p}$.

SOIL SURVEY LABORATORY STAFF, 1996. Soil Survey Laboratory Methods Manual. Soil Survey Investigations Report, vol. 42. USDA SCS, Washington DC, USA.
SOUCHIE, E.L., AZCÓN, R., BAREA, J.M., SAGGIN-JÚNIOR, O.J., RIBEIRO DA SILVA, E.M., 2006. Phosphate solubilization and synergism between P-solubilizing and arbuscular mycorrhizal fungi. Pesq. Agrop. Bras. 41(9): 1405-1411.

STEUBING, L., GODOY, R., ALBERDI, M., 2002. Métodos de ecología forestal. Universidad Austral de Chile. Editorial Universitaria. Santiago, Chile, 345 p.

TABATABAI, M.A., 1994. Soil enzymes. In: Methods of Soil Analysis, Part 2, Microbiological and Biochemical Properties. Soil Sci. Soc. Am. Madison, p. 775-833.

TABATABAI, M.A., BREMNER, J.M., 1969. Use of $p$-nitrophenyl phosphate for assay of soil phosphatase activity. Soil Biol. Biochem. 1: 301-307.

TADANO, K., 1993. Secretion of acid phosphatase by the roots of crop plants under phosphorus-deficient properties of the enzyme secreted by lupin roots. Plant Soil 155/156: 95-98.

VAN DER HEIJDEN, M.G.A., BOLLER, T., WIEMKEN, A., SANDERS, I.R., 1998. Different arbuscular mycorrhizal fungal species are potential determinants of plant community structure. Ecology 19: 2082-2091. 